La Revue

des Droits

de l'Homme

\section{La Revue des droits de l'homme}

Revue du Centre de recherches et d'études sur les droits fondamentaux

Actualités Droits-Libertés | 2018

\title{
Ainsi parlait Daoudi, une jurisprudence pour tous et pour personne
}

Interdiction de la torture et droit de requête individuelle (Article 3 et 34

Conv.EDH

\author{
Almodis Peyre
}

\section{OpenEdition}

\section{Journals}

Electronic version

URL: http://journals.openedition.org/revdh/3854

DOI: 10.4000/revdh.3854

ISSN: 2264-119X

\section{Publisher}

Centre de recherches et d'études sur les droits fondamentaux

\section{Electronic reference}

Almodis Peyre, "AINSI PARLAIT DAOUdI, UNE JURISPRUDENCE POUR TOUS ET POUR PERSONNE », La Revue des droits de l'homme [Online], Actualités Droits-Libertés, Online since 18 May 2018, connection on 01 May 2019. URL : http://journals.openedition.org/revdh/3854; DOI : 10.4000/revdh.3854

This text was automatically generated on 1 May 2019.

Tous droits réservés 


\title{
Ainsi parlait Daoudi, une jurisprudence pour tous et pour personne
}

Interdiction de la torture et droit de requête individuelle (Article 3 et 34 Conv.EDH

\author{
Almodis Peyre
}

1 La Cour européenne a rendu le 1er février 2018 sa décision quant à l'expulsion de M.A, condamné en France pour terrorisme, vers l'Algérie. Alors qu'un tel renvoi a pu constituer selon le requérant une violation de l'article 3 de la Convention mais aussi de l'article $34 \mathrm{du}$ fait de son exécution expresse, l'intérêt majeur de cette affaire réside davantage dans le raisonnement juridique porté par la Cour que dans ses conclusions. En effet, il apparaît au fil des lignes une référence constante à l'affaire Daoudi, dont les faits de l'espèce sont fortement similaires, faisant de cette jurisprudence un modèle en matière d'expulsion vers l'Algérie. Par suite, s'établit peu à peu une véritable présomption de pays non-sûr pour cet Etat. Jurisprudence certes protectrice des droits des ressortissants de pays tiers, et néanmoins critiquable, l'affaire M.A contre France ne manque pas de contradictions et d'audace dont les origines tiennent sûrement à l'aspect purement symbolique du constat de violation de la Convention. Ainsi, reprenant tel un leitmotiv les conclusions du 3 décembre 2009, la jurisprudence M.A contre France et à travers elle l'affaire Daoudi, apparaissent comme une protection pour tous, mais surtout, et en l'espèce, pour personne.

Les faits sont déterminants pour bien comprendre et cerner les enjeux et problématiques liés à l'affaire M.A contre France sur laquelle la CEDH s'est prononcée le 1er février 2018. En 1992, le requérant rejoignit les mouvements islamistes combattant les autorités algériennes lors de la guerre civile. Recherché par les autorités judiciaires, M.A quitta l'Algérie pour se réfugier en Espagne puis en France, avant de se marier religieusement en 2004 avec une ressortissante française. Cependant, le 14 juin 2006 la justice française condamna l'intéressé à sept ans d'emprisonnement ferme ainsi qu'à l'interdiction définitive du territoire français ${ }^{1}$ au titre de peine complémentaire. Ces peines furent 
prononcées en raison de la participation de l'intéressé à une association de malfaiteurs en vue de la préparation d'actes de terrorisme notamment en Algérie et en France. Si le requérant ne fit pas appel de cette décision, il demanda néanmoins à la Cour européenne une mesure provisoire sur le fondement de l'article 39 de son règlement le 19 avril 2010, lorsque la procédure de mise à exécution de l'interdiction définitive du territoire français fut lancée. En outre, le 11 août 2011, le requérant forma une requête en relèvement de la mesure d'ITF. Cette requête fut rejetée le 18 mai 2011 par le tribunal correctionnel de Paris ; M.A. n'interjeta pas appel.

3 Le 1er juillet 2014, la Cour européenne déclara la requête aux fins de mesures provisoires du requérant irrecevable pour non-épuisement des voies de recours internes. En réponse à la notification par le préfet le 4 novembre 2014 qu'il entendait procéder à l'expulsion du requérant, M.A déposa une demande d'asile auprès de l'OFPRA le 2 décembre 2004. Cette demande d'asile fut alors étudiée selon une procédure accélérée avant d'être rejetée le 17 février 2015. Néanmoins, le requérant ne se vit notifier cette décision que le 20 février 2015 à $9 \mathrm{~h} 20$ alors qu'il se rendait au commissariat. On lui notifia par la même occasion la décision du préfet de fixer l'Algérie comme pays de renvoi. Son avocate saisit alors à nouveau la Cour d'une demande de mesures provisoires ce même jour 20 février 2015 à 15h16. A 15h54, le greffe de la Cour envoya un courrier au gouvernement français afin de suspendre l'expulsion du requérant. À 16h02, le greffe de la Cour publia les documents officiels sur le site sécurisé. Cependant, lorsque les services de police chargés du renvoi de M.A prirent connaissance de cette décision, les portes de l'avion à bord duquel se trouvait le requérant étaient déjà closes et l'avion décolla à 16h15. Dès son arrivée en Algérie, ce 20 février 2015, le requérant fut placé en garde à vue, dans un lieu tenu secret, par le Département du Renseignement et de la Sécurité (DRS) algérien.

4 Au vu des faits retranscrits, le plaignant allègue que son renvoi en Algérie constituait une violation de l'article 3 de la Convention, puisqu'il risquait une fois sur place de se voir infliger des traitements prohibés par cette disposition. En outre, M.A fait grief au gouvernement français d'avoir nié son droit de recours devant la Cour européenne, en le privant le 20 février 2015 de toute possibilité de saisir les juges strasbourgeois du fait de la rapidité de la mise à exécution de la mesure d'expulsion. Bien que la Cour ait déjà eu à trancher des affaires similaires, l'aspect politique de ce différend est majeur étant donné que le requérant fut condamné pour des actes liés au terrorisme, sujet ô combien sensible pour les Etats et leur société civile. A ce titre, certains journalistes ont pu conclure qu'« on ne peut demander la protection des valeurs démocratiques et des principes fondamentaux que l'on récuse $»^{2}$. Si la Cour ne prend heureusement pas en compte cet argument au sein de ses développements, elle rappelle néanmoins à maintes reprises la nécessité pour les Etats de tout mettre en place pour lutter contre les mouvances djihadistes. Cependant, au fil d'une jurisprudence certes critiquable sous bien des aspects, de surcroît plus symbolique qu'effective, la Cour développe une présomption de pays non-sûr à l'encontre de l'Algérie. En outre, les juges condamnent la France pour avoir réduit la protection offerte par la Convention à M.A en préparant les modalités nécessaires à son renvoi, en amont de la notification au requérant de son expulsion vers l'Algérie,. Si la recevabilité de l'article 3 est discutable mais prévisible du fait même des jurisprudences antérieures de la Cour (I), la violation de cette disposition repose sur une situation inchangée en Algérie depuis l'affaire Daoudi de 2009 (II), situation qui n'empêcha pourtant pas le gouvernement français d'expulser l'intéressé dans un délai ne lui permettant pas de faire valoir ses droits (III). 


\section{L'ÉPUISEMENT DES VOIES DE RECOURS INTERNES : UN LABYRINTHE JURISPRUDENTIEL}

5 La question centrale posée à la Cour concernant l'épuisement des voies de recours internes est celle de savoir si l'absence de recours contre les décisions de refus d'asile par l'OFPRA ${ }^{3}$, de désignation de l'Algérie comme pays de retour par le préfet, ou d'interdiction du territoire prononcée par le tribunal correctionnel, peut constituer un non-respect de l'article 35 de la Convention. Pour répondre à cette question, la Cour reprend une jurisprudence constante rappelant que seuls les recours effectifs, en l'occurrence suspensifs de la décision de renvoi, doivent être pris en compte dans l'épuisement des voies de recours internes (A). Néanmoins, cette exigence peut en l'espèce conduire à une conclusion critiquable, puisque guidée par une analyse antérieure des faits (C), quitte à omettre certaines possibilités pourtant ouvertes (B).

\section{A.L'OBLIgATION D'ÉPUISER UNE VOIE DE RECOURS SUSPENSIVE}

6 Pour donner suite à la contestation par le gouvernement du respect de l'article 35 de la Convention, la Cour rappelle dans un premier temps que l'obligation d'épuiser les voies de recours internes ne s'applique que lorsque «l'ordre interne offre un recours effectif quant à la violation alléguée $»^{4}$. Cette obligation d'épuisement des voies de recours dits effectifs repose aujourd'hui sur une jurisprudence constante. On peut citer à titre d'exemple les affaires Selmouni c. France ou encore McFarlane c. Irlande dans lesquelles ont été considérés comme effectifs les recours " à la fois relatifs aux violations incriminées, disponibles et adéquats " qui offraient aux demandeurs des possibilités d'introduire des actions contre des mesures leur faisant grief « à un degré suffisant de certitude non seulement en théorie mais aussi en pratique $»^{5}$.

7 Or, dans le cas d'un renvoi d'une personne de la part d'un pays partie à la Convention vers un Etat tiers, les recours dits effectifs sont ceux qui ont un effet suspensif. Cette nécessité du caractère suspensif du recours contre l'exécution d'une mesure d'expulsion a posée par la Commission européenne des droits de l'Homme, notamment dans sa décision du 26 mai 1976, dans laquelle elle a pu déclarer que les recours « ne [pouvaient], en l'espèce, être considérés comme efficaces [...] la procédure relative à l'asile politique [n'ayant] pas, en elle-même, d'effet suspensif de la mesure d'expulsion $»^{6}$. Cette exigence fut ensuite réitérée à maintes reprises notamment dans l'affaire Sultani c. France où la Cour - cette fois-ci - déclara que « lorsqu'un individu se plaint de ce que son renvoi l'exposerait à un traitement contraire à l'article 3 de la Convention, les recours sans effet suspensif ne peuvent être considérés comme efficaces au sens de l'article $35 \$ 1$ de la Convention », puisque « compte tenu de la nature irréversible du dommage susceptible d'être causé en cas de réalisation d'un risque de torture ou de mauvais traitements, la notion de recours effectif au sens de l'article 13 requiert la possibilité de faire surseoir à l'exécution d'une mesure d'expulsion $»^{7}$. En somme, seul « un contrôle juridictionnel, lorsqu'il existe et lorsqu'il fait obstacle au renvoi, doit être considéré comme un recours effectif $»^{8}$.

8 En l'espèce, la Cour prend soin de démontrer qu'aucun des recours que M.A pouvait introduire contre la décision de renvoi en Algérie n'avait les qualités requises pour être considéré comme effectif. D'une part, le fait pour l'OFPRA d'avoir eu recours à une 
procédure d'asile prioritaire pour examiner la demande du requérant engendra l'absence de recours suspensif devant la CNDA, contrairement à ce qui était alors prévu pour les procédures dites normales'. D'autre part, pour ce qui est de l'arrêté désignant l'Algérie comme pays de destination, la Cour précise qu'un recours contre cette décision ne pouvait acquérir la qualité de recours suspensif que s'il avait été combiné avec un recours contre la mesure d'interdiction du territoire ${ }^{10}$. Or, cette mesure d'interdiction étant devenue définitive au moment de la saisine de la Cour, aucun recours suspensif contre la décision du préfet ne pouvait être mis en œuvre par M.A. Ainsi, bien que le requérant n'ait jamais contesté la décision de l'OFPRA ou celle du préfet, ce dernier a bel et bien épuisé toutes les voies de recours suspensives, et donc effectives, qui lui étaient offertes. Il convient de préciser qu'une telle conclusion ne serait à ce jour plus permise puisque la distinction entre les deux types de procédures devant l'OFRA n'a plus d'incidence sur le caractère suspensif du recours. En effet, la loi française $n^{\circ} 2015-925$ du 29 juillet 2015 relative à la réforme du droit d'asile a permis de systématiser le recours suspensif devant la CNDA, quelle que soit la procédure utilisée. Toutefois, du fait des législations en vigueur au moment des faits, la condition de saisine de la Cour mentionnée à l'article 35 de la Convention ${ }^{11}$ ne peut faire obstacle aux griefs formulés par le requérant.

9 En outre, la Cour ajoute à son argumentation qu'un requérant qui a utilisé une voie de recours effective mais sans toutes les épuiser ne peut se voir reprocher ce fait si les autres voies de recours en jeu n'avaient pas plus de chance d'aboutir ${ }^{12}$. Etablissant un parallèle avec l'affaire Y.P et L.P contre France, la Cour explique qu'on ne peut reprocher à M.A de ne pas avoir intenté un recours contre l'arrêté du préfet désignant l'Algérie comme pays de renvoi puisqu' avait été rejetée sa demande d'asile, étudiant déjà la compatibilité de ce renvoi avec l'article 3 de la Convention,. Or, un pourvoi contre l'arrêté n'aurait eu que peu de chance d'aboutir, la situation en Algérie n'ayant guère évolué entre les deux décisions. En définitive, seul compte le fait que le requérant ait pu poursuivre une voie de recours effective, permettant aux autorités juridictionnelles nationales de vérifier si son expulsion ne comportait pas un risque qu'il soit soumis à des traitements inhumains ou dégradants. Il importe peu que le demandeur ait intenté une voie de recours précise, la Cour ne pouvant déterminer qu'une voie de recours serait plus opportune qu'une autre.

\section{B. L'OBJET DU RECOURS CONTRE UNE DÉCISION D'ITF : QUID DE LA JURISPRUDENCE DAOUDI?}

10 Si les conclusions de la Cour quant aux possibles recours contre les décisions de l'OFPRA ou du préfet sont somme toute dans la droite ligne d'une jurisprudence bien établie, il est néanmoins possible de remettre en cause le raisonnement tenu par les juges quant à l'analyse de la décision d'ITF. En l'espèce, la Cour qualifie un éventuel recours contre la décision du tribunal correctionnel de Paris du 14 juin 2006 comme n'ayant pour seul objet que de vérifier l'opportunité d'une mesure d'interdiction du territoire, ce qui le rendrait donc inutile. Pour ce faire, la Cour rappelle qu'un recours contre l'interdiction définitive du territoire français n'est pas nécessaire pour considérer qu'il y a épuisement des voies de recours internes, ce dernier n'ayant d'autre objectif que de réévaluer l'opportunité de cette interdiction et non de déterminer vers quel pays l'intéressé aurait pu être renvoyé. N'analysant pas l'opportunité d'un renvoi vers un pays déterminé, le recours contre la mesure d'ITF n'aurait alors pu permettre de contrôler le respect des obligations posées par l'article 3. C'est donc, selon la Cour, l'objet même du recours qui justifie 
premièrement l'irrecevabilité de l'allégation de violation du droit au respect de la vie privée et familiale et deuxièmement la recevabilité de l'allégation de violation de l'article 3.

11 Cette conclusion semble omettre dans une certaine mesure la jurisprudence antérieure de la Cour. En effet, les juges européens ont pu souligner dans la jurisprudence Daoudi c. France que la Cour d'appel de Paris dans son arrêt du 24 novembre 2008 avait bel et bien étudié la compatibilité d'une mesure d'interdiction du territoire avec les articles 8 et 3 de la Convention ${ }^{13}$. Ainsi, aurait été prise en compte non seulement la question de l'opportunité de la mesure d'interdiction du territoire, mais aussi celle du choix du pays de renvoi. Or, la Cour ne pouvait vraisemblablement ignorer sa jurisprudence antérieure du fait même que cette décision est citée près de 17 fois dans l'affaire en cause et qu'elle constitue en outre le fondement de la violation sur le fond de l'article 3. Ainsi, il aurait été tout à fait possible qu'un recours en appel contre la décision d'ITF de 2006 puisse avoir pour objet, non pas unique mais auxiliaire, d'étudier l'éventualité d'une violation de l'article 3 de la Convention par le maintien de cette mesure d'éloignement du territoire.

\section{C.UNE DEMANDE D'ASILE FRAUdULEUSE MAIS NÉCESSAIRE}

La Cour ne peut conclure en la violation de l'article 35.1 - quitte à passer sous silence la jurisprudence Daoudi - du fait même de sa précédente décision de 2014. En effet, dans l'affaire M.X contre France, dans laquelle le requérant avait fait une demande de mesure provisoire en vertu de l'article 39 du règlement de la Cour, les juges de Strasbourg ont pu conclure "que le requérant, en omettant d'introduire une demande d'admission au bénéfice de l'asile auprès de l'OFPRA, [s'était] abstenu de faire usage d'une voie de recours effective $~^{14}$. La Cour a donc indiqué qu'une demande d'asile aurait permis le cas échéant de conclure à l'épuisement des voies de recours effectifs. De la sorte, elle a conditionné indirectement l'admission d'une future requête à la saisine de l'OFPRA, qui lorsqu'elle fut faite, ne pouvait que lier les juges. Or, comme le souligne la juge O'LEARLY, cette interprétation contrevient au principe même selon lequel il n'appartient en aucun cas à la Cour « d'affirmer qu'une voie de droit serait, à l'égard du requérant, plus opportune qu'une autre $»^{15}$.

13 Le raisonnement tenu par la Cour dans son affaire M.A c. France - de même que dans sa décision M.X c. France -, peut tout autant être tenu pour critiquable dans la mesure où une demande d'asile, dans ces circonstances, aurait pu être considérée comme frauduleuse. En effet, toujours selon la jurisprudence de la Cour, « une demande est considérée comme reposant sur une fraude délibérée ou constituant un recours abusif lorsque, par exemple, le demandeur dépose plusieurs demandes d'asile sous des identités différentes, lorsqu'il formule une telle demande lors de la notification d'une mesure d'éloignement ou lors d'une interpellation alors qu'il se trouve en France depuis un certain temps ${ }^{16}$. L'utilisation de la conjonction de coordination «ou » et du terme «par exemple» montre bien que ces diverses conditions ne sont pas cumulatives ni exhaustives. La demande d'asile déposée par M.A le 2 décembre 2014, « en réponse $»^{17}$ à la notification de sa mesure d'expulsion le 4 novembre 2014, aurait donc pu être qualifiée de frauduleuse.

14 Si on s'en tient au critère suspensif conditionnant l'effectivité des voies de recours, les conclusions de la Cour paraissent conformes aux précédents jurisprudentiels, car ni le recours devant la CNDA ni le recours contre l'arrêté préfectoral n'étaient en l'occurrence 
suspensifs. Ce qui demeure dommageable tient au raisonnement appliqué en l'espèce à la mesure d'interdiction du territoire, et à l'origine de ce raisonnement qui est à trouver dans l'arrêt de M.X c. France 2014. En concluant à l'obligation de demander l'asile pour remplir les critères posés par l'article 35 , les juges ont omis d'une part le fait que cette demande d'asile puisse être considérée comme frauduleuse, d'autre part qu'un appel contre la décision d'ITF de 2006 aurait pu amener à contrôler le choix du pays de destination en vertu des obligations issues de l'article 3.

\section{Expulsion d'une personne condamnée pour terrorisme vers l'Algérie : l'affaire Daoudi, un arrêt fondateur?}

15 Une fois la recevabilité de l'allégation de violation de l'article 3 reconnue, la Cour peut engager la responsabilité d'un Etat «lorsqu'il y a des motifs sérieux et avérés de croire que l'intéressé, si on l'expulse vers le pays de destination, y courra un risque réel d'être soumis à un traitement contraire à l'article $3 \|^{18}$, lorsque « l'obligation de ne pas expulser la personne en question vers ce pays $»^{19}$ n'a pas été respectée. La cour devra pour cela examiner "les conséquences prévisibles du renvoi du requérant dans le pays de destination, compte tenu de la situation générale dans celui-ci et des circonstances propres au cas de l'intéressé $»^{20}$. Dans l'application de ces conditions au cas d'espèce, il apparaît que la Cour affirme une présomption de pays non-sûr envers l'Algérie (A), tout en déterminant la situation personnelle du requérant par analogie avec l'affaire Daoudi (B), quitte à subsister sa propre vision des faits à celle des tribunaux internes (C).

\section{A.Une présomption de pays non-sûr pour l'Algérie}

En ce qui concerne la situation générale en Algérie et pour comprendre si le renvoi du requérant dans ce pays constituait un risque sérieux de traitement inhumain et dégradant, la Cour fait référence à plusieurs rapports externes. D'abord, elle mentionne ses conclusions dans l'affaire Daoudi, quant aux traitements infligés en détention aux personnes soupçonnées de participer à des actes de terrorisme. Elle rappelle ensuite les conclusions du groupe de travail du Comité des droits de l'homme des Nations Unies dans son rapport sur l'Algérie de 2012. Enfin, elle invoque les rapports de 2014 et de 2015 d'Amnesty international ${ }^{21}$ et la contribution d'Human Rights Watch en 2016 quant à l'examen périodique universel de l'Algérie par le Conseil des droits de l'homme ${ }^{22}$. La cour spécifie qu'il s'agit bien de la situation au 20 février 2015, jour de l'expulsion du requérant, qu'il convient de prendre en considération pour examiner si M.A courait un risque d'être soumis à un traitement prohibé par l'article 3 lorsqu'il fut renvoyé vers l'Algérie. Les juges mentionnent par la suite le fait qu'ils n'ont été saisis «d'aucun élément relatif à l'évolution de la situation en Algérie depuis l'adoption de l'arrêt Daoudi, de nature à remettre en cause l'appréciation des faits à laquelle [ils se sont livrés] dans cette affaire ${ }^{23}$. Les divers rapports susmentionnés et postérieurs à l'affaire Daoudi, n'ont eu de cesse que de confirmer des faits qui atteignent « sans conteste le seuil requis pour l'application de l'article 3 de la Convention $»^{24}$. La Cour se fonde ainsi sur des rapports antérieurs mais aussi postérieurs au renvoi de M.A pour déterminer le cadre général de la détention en Algérie. Ce cadre général n'ayant en l'espèce pas évolué depuis la 
jurisprudence de 2009, il convient de conclure pour les juges de Strasbourg à l'existence du seuil requis pour l'application de l'article 3. que deux autres personnes condamnées en France pour les mêmes faits que M.A, et renvoyés en Algérie, n'ont jamais mentionné avoir subi un comportement contraire à l'article 3 de la part des autorités algériennes. Pour ce faire, les juges rappellent que « la Cour ne saurait déduire de ces seules allégations [...] que le requérant ne serait pas, personnellement, soumis à un risque de subir des traitements prohibés par l'article 3 de la convention en cas de retour en Algérie $»^{26}$. De ce fait, la Cour tend à laisser penser que, si les allégations du requérant quant au risque de violation de l'article 3 peuvent être étayées par des rapports généraux et non personnellement attribuables à son cas, les réponses des gouvernements dans le but de dissiper ces doutes doivent, elles, être parfaitement circonstanciées. Si la présomption de pays non-sûr planant sur l'Algérie est réfragable, elle ne pourra être renversée que par le biais de preuves circonstanciées portant sur la situation personnelle du requérant.

\section{B.La situation personnelle du requérant : un risque déterminé par analogie...}

19 En ce qui concerne le cas personnel de M.A, la Cour souligne que le requérant a été condamné pour des faits de participation à une association de malfaiteurs en vue de la préparation d'actes de terrorisme, tout comme Kamel Daoudi. Cette décision a été rendue publique et demeure donc potentiellement connue par les autorités algériennes. Le risque est en l'espèce confirmé par le fait que le requérant a été appréhendé dès son arrivée en Algérie par le Département du Renseignement et de la Sécurité (DRS), avant d'être soumis à une détention au secret. Ainsi, et parce que le Gouvernement français ne peut produire des éléments réfutant ces faits, la Cour conclut que le requérant au moment de son renvoi fut bel et bien exposé à un risque réel et sérieux de se voir infliger des traitements contraires à l'article 3 , et que de ce fait cette disposition a été violée par la République française.

Or, c'est sur la preuve de ce danger du fait de la situation personnelle de l'intéressé que la juge O'LEARY porte la plus grande critique. Selon elle, la Cour en fonctionnant par analogie avec l'affaire Daoudi n'a pas tenu de raisonnement suffisamment développé et prudent. Notamment, il serait possible de douter de la "notoriété » du requérant dans son pays d'origine. Comme le souligne la juge, l'identité du requérant a toujours été cachée par ce dernier, utilisant pour ce faire divers patronymes. En outre, elle reproche à la Cour de ne pas faire de distinction « entre les cas où les intéressés ont fait l'objet de poursuites judiciaires et/ou de condamnations en Algérie, de sorte qu'ils sont 
individualisés ou visés dans ce pays, et ceux où l'existence de poursuite judiciaire n'est pas établie ${ }^{27}$. Elle ajoute que, si le requérant a bien été détenu dans un lieu tenu secret du 20 février au 3 mars 2015, il n'existe aucune preuve matérielle attestant d'actes contraires à l'article 3 à l'encontre de M.A. En somme, selon la juge O'LEARY, «l'arrêt semble créer - par inadvertance ou non - un obstacle à des expulsions vers l'Algérie pour des raisons générales sans exiger un examen individuel des circonstances de la personne intéressée $»^{28}$.

Si on peut adhérer à l'opinion de la juge O'LEARY quant à la question de l'épuisement des voies de recours internes, il semble nécessaire de prendre ici quelques distances avec le raisonnement déployé par elle. En ce qui concerne l'absence d'identification certaine de l'intéressé, ce doute ne saurait lever le risque de traitements contraires à l'article 3. Rappelons que le requérant fit l'objet d'une décision de condamnation pour participation à une association de malfaiteurs en vue de la préparation d'actes de terrorisme, décision largement motivée, détaillée, rendue publique, donc accessible par les autorités algériennes. Ainsi, et dans ces conditions, il n'est pas souhaitable de faire une distinction entre les cas où l'intéressé aurait fait l'objet de poursuites dans le pays de renvoi et les cas où l'existence de ces poursuites n'est pas avérée, au risque sinon de nier l'utilité de la jurisprudence Soering ${ }^{29}$. Cette dernière a pour but de prévenir tout risque de traitements inhumains et dégradants, ce risque n'ayant pas besoin pour exister de poursuites judiciaires ni même de pouvoir être sûr de l'identité de la personne. Une telle distinction permettrait aux Etats ayant recours à des actes prohibés par l'article 3 de se voir ouvrir une nouvelle possibilité de "récupérer » un de leurs nationaux s'ils attendent l'expulsion de ce dernier pour ouvrir contre lui des poursuites judiciaires.

En somme, malgré les zones d'ombre quant à l'identité du requérant ou même le fait qu'il n'est pas avéré qu'il risquait de subir des poursuites judiciaires de la part des autorités algériennes, il existait bel et bien, au moment de son renvoi, un risque réel de traitements prohibés par la Convention européenne. Si ce risque, de par sa définition même, suppose une certaine éventualité non vérifiable et non vérifiée, c'est, « l'absence de certitudes sur son sort en cas d'expulsion et l'impossibilité de contrôler ce qu'il pourrait subir une fois sur le sol algérien [qui] conduit la Cour à $»^{30}$ conclure en une violation de l'article 3. La critique que l'on peut faire à la Cour ne se situe pas sur le raisonnement juridique l'ayant conduit à conclure à la violation de l'article 3 par la France, mais porte sur le fait que cette conclusion ait pour conséquence une substitution de sa propre vision des faits à celle des organes français.

\section{C. ...Quitte à substituer sa propre vision des faits à celle des tribunaux internes}

23 Ce qui pourrait être reproché à la Cour, et qui ressort notamment dans l'opinion séparée de la juge O'LEARY, concerne l'analyse proposée par les juges de la décision de l'OFPRA. Pour évincer l'argument du gouvernement selon lequel le requérant a attendu près de 15 ans pour demander l'asile en France, la Cour rappelle que l'OFRA «mieux placé pour apprécier non seulement les faits, la crédibilité de témoins, ainsi que le comportement de la personne [...] n'a nullement pris cette circonstance en compte dans sa décision $»^{31}$. En plaçant l'OFPRA comme une organisation plus apte qu'elle à étudier la situation concrète des demandeurs d'asile, la Cour fait naître un véritable paradoxe. En effet, les juges se justifient du fait de ne pas prendre en compte le délai de 15 ans entre l'arrivée du 
requérant et sa demande d'asile derrière le fait que l'OFPRA en a fait de même. Or, l'office contrairement à la Cour, et comme le souligne le gouvernement, a en outre conclu en l'absence de risque de violation de l'article 3 de la convention compte tenu précisément de la situation du requérant. Si l'OFPRA est donc plus habilité que la Cour de Strasbourg pour conclure que le délai de 15 ans ne doit pas être pénalisant pour le requérant, il l'est en somme moins pour déterminer le risque de violation de l'article 3 eu égard à la situation matérielle de M.A.

Cette conclusion apparaît alors comme critiquable au vu de la jurisprudence antérieure de la Cour, qui a notamment pu rappeler qu'« en principe, là où des procédures internes ont été menées, ce n'est pas la tâche de la Cour de substituer sa propre version des faits à celle des juridictions nationales, auxquelles il appartient d'établir les faits sur la base des

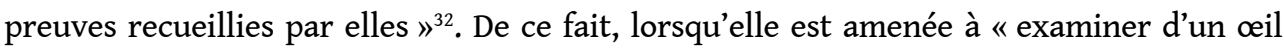
plus critique les conclusions des juridictions nationales [...] il lui faut néanmoins disposer d'éléments convaincants pour pouvoir s'écarter des constatations auxquelles celles-ci sont parvenues $»^{33}$, éléments convaincants dont la Cour ne se justifie point. Néanmoins, il est possible d'expliquer cette mise à l'écart des analyses de l'OFPRA par le fait que cette dernière avait eu recours à une procédure accélérée pour étudier la demande d'asile de M.A, procédure dont on connaît les lacunes et le caractère quelque peu expéditifif ${ }^{34}$.

\section{Embarquement immédiat sans retour possible : une condamnation de principe}

Enfin, à la suite de la notification au requérant du refus de sa demande d'asile par l'OFPRA et de la désignation de l'Algérie comme pays de destination, son avocate décida le 20 février 2015 à 15 h16 de saisir la Cour d'une demande de mesure provisoire. La Cour confirma alors à $15 \mathrm{~h} 54$ au gouvernement l'application de l'article 34 de la convention dans cette affaire, décision publiée à 16 h02 sur le site sécurisé. M.A fut néanmoins renvoyé vers l'Algérie par avion à $16 \mathrm{~h} 15$. Ce renvoi priva d'une part l'intéressé de sa possibilité de saisir la Cour de manière efficace et d'autre part une condamnation de la France toute effectivité.

26 "Dans le système de la Convention, les mesures provisoires [...], se révèlent d'une importance fondamentale pour éviter des situations irréversibles qui empêcheraient la Cour de procéder dans de bonnes conditions à un examen de la requête et, le cas échéant, d'assurer au requérant la jouissance pratique et effective du droit [...] qu'il invoque. Dès lors, dans ces conditions, l'inobservation par un Etat défendeur de mesures provisoires met en péril l'efficacité du droit de recours individuel, tel que garanti par l'article $34 \|^{35}$. Ainsi, dans le cadre de l'examen d'un grief au titre de l'article 34 de la Convention, le gouvernement doit prouver " que la mesure provisoire a été respectée ou, dans des cas exceptionnels, qu'il y a eu un obstacle objectif qui l'a empêché de s'y conformer " ${ }^{36}$. Néanmoins, l'Etat devra avoir "entrepris toutes les démarches raisonnablement envisageables pour supprimer l'obstacle et pour tenir la Cour informée de la situation $»^{37}$. De ce fait, la question qui est ici posée à la Cour est celle de savoir si le bref laps de temps entre la notification de l'application de l'article 34 et le décollage de l'avion de M.A peut constituer un obstacle objectif à la réalisation et au respect de cet article.

Aux yeux de la Cour, les autorités françaises elles-mêmes ont créé les conditions amenant le requérant à ne pouvoir que difficilement saisir la Cour d'une mesure provisoire. En effet, la décision de l'OFPRA bien que prise le 17 février 2015 ne fut notifiée au requérant 
que le 20 février à l'occasion de son pointage au commissariat. Le même jour, on l'informa de la décision de renvoi vers l'Algérie. Or, à la date de cette notification « les services de police avaient déjà fixé les modalités retenues pour le transport du requérant à la frontière "; et "dès le 19 février 2015, les autorités consulaires algériennes avaient délivré, à l'insu du requérant, un laissez-passer à la demande des autorités françaises ${ }^{38}$. $\mathrm{Du}$ fait de ces préparatifs, le renvoi de M.A put avoir lieu seulement sept heures après sa notification. De ce bref délai entre la notification du renvoi et son exécution, la Cour conclut que « les autorités françaises ont créé des conditions dans lesquelles le requérant ne pouvait que très difficilement saisir la Cour d'une seconde demande de mesure provisoire $\aleph^{39}$. Se prévaloir de ce délai pour justifier l'inexécution de la mesure provisoire reviendrait alors à nier l'adage " nemo auditur propriam suam turpitudinem allegans ». Le gouvernement, en préparant au préalable l'expulsion de M.A ., a « délibérément et de manière irréversible, amoindri le niveau de protection des droits énoncés dans l'article 3 de la Convention que le requérant cherchait à faire respecter en introduisant sa demande devant la Cour $»^{40}$, violant ainsi l'article 34 de la Convention et privant le requérant de toute protection effective.

En outre, et du fait que l'Algérie n'est pas partie à la Convention européenne, le renvoi exprès du requérant vers ce pays « a pour le moins ôté toute utilité à l'éventuel constat de violation de la Convention $»^{41}$. Comme la Cour le rappelle, le gouvernement français doit « entreprendre toutes les démarches possibles pour obtenir des autorités algériennes l'assurance concrète et précise que le requérant n'a pas été et ne sera pas soumis à des traitements contraires à l'article 3 de la Convention $\star^{42}$. Mais il ne s'agit là que d'une obligation de moyen.

Bien que la France soit condamnée par la Cour pour violation des articles 3 et 34 de la Convention, il est très probable que la situation du requérant demeure inchangée. Ce constat pose une nouvelle fois le difficile problème de l'exécution extraterritoriale des arrêts de la Cour, exécution qui demeure soumise à des considérations plus diplomatiques que juridiques. En somme, il est alors nécessaire de constater que cette affaire repose plus sur forme de satisfaction équitable que de véritable protection des droits de l'homme, donnant de ce fait aux dispositions de l'arrêt une portée moins effective que symbolique.

Cour. EDH, 5e section, 1er février 2018, M.A contre France, req. n 9373/15

Les Lettres "Actualités Droits-Libertés 》 (ADL) du CREDOF (pour s'y abonner) sont accessibles sur le site de la Revue des Droits de l'Homme (RevDH) - Contact

\section{NOTES}

1. Cette interdiction sera désormais nommée sous le nom de mesure d'ITF (Interdiction du Territoire Français).

2. Petrilli, «Terrorisme islamique : la CEDH hors sol », Marianne [en ligne], mis en ligne le 28 février 2018, consulté le 12 mars 2018. URL: https://www.marianne.net/debattons/ forum/terrorisme-islamique-la-cedh-hors-sol 
3. Office Français de Protection des Réfugiés et Apatrides (OFPRA).

4. CEDH, sect. V, $1^{\text {er }}$ février 2018, M.A contre France, req. $n^{\circ} 9373 / 15$, $\$ 39$.

5. CEDH, GC, 28 juillet 1999 , Selmouni $c$. France, req. $n^{\circ} 25803 / 94 \$ 75$. Voir en outre CEDH, GC, 10 septembre 2010, McFarlane c. Irlande, req. $n^{\circ} 31333 / 06, \$ 107$.

6. Com.EDH, 20 mai 1976, X. c/république fédérale d'Allemagne, req. $\mathrm{n}^{\circ}$ 7216/75.

7. CEDH, sect. III, 20 septembre 2007, Sultani c. France, req. $n^{\circ} 45223 / 05$, $\$ 50$.

8. CEDH, sect. V, 1er février 2018, M.A contre France, req. $n^{\circ} 9373 / 15$, $\$ 41$.

9. Pour une analyse des risques des procédures accélérées de demande d'asile voir Nicolas Hervieu, « Droit français de l'asile et procédure prioritaire : de l'art français d'ouvrir les yeux à Strasbourg (CEDH, Anc. 5e Sect. 2 février 2012, I.M. c. France) », Combats pour les droits de l'homme (CPDH) [En ligne], mis en ligne le 3 février 2012, consulté le 09 mars 2018. URL: http://combatsdroitshomme.blog.lemonde.fr/2012/02/03/droit-francais-de-1 \%Е2\%80\%99asile-et-procedure-prioritaire-de-l\%E2\%80\%99art-francais-d\%E2\%80\% 99ouvrir-les-yeux-a-strasbourg-cedh-anc-5e-sect-2-fevrier-2011-i-m-c-france/ 10. Voir article L513-3 du CESEDA disposant que : «Le recours contentieux contre la décision fixant le pays de renvoi n'est suspensif d'exécution, dans les conditions prévues au second alinéa de l'article L. 512-3, que s'il est présenté en même temps que le recours contre la mesure d'éloignement qu'elle vise à exécuter ».

11. La cour a déjà pu arriver à de telles conclusions quant à des recours devant l'OFPRA ou devant le juge administratif dans l'affaire $\mathrm{CEDH}$, sect. V, 18 novembre 2010, Boutagni $c$. France, req. $\mathrm{n}^{\circ} 42360 / 08, \S 35-37$. Pour une analyse plus complète de cette jurisprudence voir Nicolas Hervieu, « Renvoi vers le Maroc d'un homme condamné en France pour terrorisme et engagement gouvernemental de non expulsion ", La Revue des droits de l'homme [En ligne], Actualités Droits-Libertés, mis en ligne le 18 novembre 2010, consulté le 09 mars 2018. URL : http://journals.openedition.org/revdh/3622 ; DOI : 10.4000/ revdh.3622

12. Le principe avait été déjà posé par la Cour dans l'affaire CEDH, GC, 29 avril 1990, Aquilina c. Malte, req. ${ }^{\circ}$ 25642/94, \$39.

13. CEDH, sect. V, 3 décembre 2009, Daoudi c. France, req. $n^{\circ}$ 19576/08, \$27

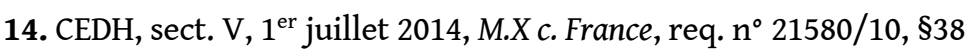

15. CEDH, sect. V, $1^{\text {er }}$ février 2018, M.A contre France, req. $n^{\circ} 9373 / 15$, $\$ 43$

16. CEDH, sect. V, 2 février 2012, I.M c. France, req. n ${ }^{\circ} 9152 / 09$, $\$ 46$

17. Terme employé par la Cour elle-même dans le paragraphe 17 de l'affaire M.A contre France.

18. Ibid., $\$ 51$.

19. Ibid., \$51. Ce principe a aujourd'hui valeur de jurisprudence constante et fut mis en place pour la première fois par la Cour en 1989 (CEDH, Cour plénière, 7 juillet 1989, Soering c. Royaume Uni, req. $n^{\circ} 14038 / 88, \S 91$ ).

20. Ibid., \$130.

21. Voir, Amnesty internationale, « Rapport 2014/15, la situation des droits humains dans le monde », mis en ligne le 25 février 2015, consulté le 3 avril 2018. URL : https:// www.amnesty.org/fr/documents/document/?indexNumber=pol10\%2F0001\% 2F2015\&language=fr. Voir en outre, Amnesty internationale, « Rapport 2015/16, la situation des droits humains dans le monde ", mis en ligne le 23 février 2016, consulté le 3 avril 2018. URL : https://www.amnesty.org/fr/documents/pol10/2552/2016/fr/ 22. Voir, Human Rights Watch, «Soumission pour l'examen périodique universel de l'Algérie », mis en ligne le 14 octobre 2016, consulté le 3 avril 2018. URL : https:// 
www.hrw.org/fr/news/2016/10/14/soumission-pour-lexamen-periodique-universel-delalgerie

23. CEDH, sect. $V$, $1^{\text {er }}$ février 2018, M.A contre France, req. $n^{\circ} 9373 / 15$, $\$ 54$.

24. CEDH, sect. $\mathrm{V}, 1^{\mathrm{er}}$ février 2018, M.A contre France, req. $\mathrm{n}^{\circ} 9373 / 15$, $\$ 54$.

25. CEDH, GC, 28 février 2008, Saadi c. Italie, req. n³7201/06, §131.

26. CEDH, sect. V, 1er février 2018, M.A contre France, req. n 9373/15, $\$ 57$ (souligné par nos soins).

27. Ibid., \$37.

28. Ibid., $\$ 38$.

29. Voir CEDH, Cour plénière, 7 juillet 1989 , Soering c. Royaume Uni, req. $\mathrm{n}^{\circ} 14038 / 88$

30. Nicolas Hervieu, «Inconventionnalité d'une expulsion vers l'Algérie d'un terroriste islamiste (CEDH 3 décembre 2009, Daoudi c. France) ", Combats pour les droits de l'homme (CPDH) [En ligne], mis en ligne le 3 décembre 2009, consulté le 09 mars 2018. URL : http://combatsdroitshomme.blog.lemonde.fr/2009/12/03/inconventionnalitedune-expulsion-vers-lalgerie-dun-terroriste-islamiste-cedh-3-decembre-2009-daoudi-cfrance/ (souligné par nos soins).

31. CEDH, sect. V, 1er février 2018, M.A contre France, req. n 9373/15, Opinion séparée du juge O'LEARY, $\$ 56$.

32. CEDH, GC, 24 mars 2011, Giuliani et Gaggio c. Italie, req. $n^{\circ} 23458 / 02$, $\$ 180$.

33. CEDH, sect. V, 16 juillet 2015, Ghedir et autres c. France, req. $n^{\circ}$ 20579/12, \$111.

34. Pour plus de détails sur les origines de cette critique, voir Yehudi Pelosi, « Une avancée incertaine pour l'effectivité des recours des demandeurs d'asile " prioritaires ", La Revue des droits de l'homme [En ligne], Actualités Droits-Libertés, mis en ligne le 07 mars 2014, consulté le 22 mars 2018. URL : http://journals.openedition.org/revdh/610 ; DOI : $10.4000 /$ revdh. 610

35. CEDH, CG, 4 février 2005, Mamatkoulov et Askarov c. Turquie, req. $\mathrm{n}^{\circ}$ 46827/99 et $46951 / 99, \$ 125$.

36. CEDH, GC, 10 mars 2009, Paladi c. Moldova, req. n 39806/05, §92.

37. Ibid.

38. CEDH, sect. V, 1er février 2018, M.A contre France, req. $n^{\circ} 9373 / 15$, $\$ 69$.

39. Ibid., $\$ 70$.

40. Ibid.

41. Ibid.

42. CEDH, sect. V, 1er février 2018, M.A contre France, req. $n^{\circ}$ 9373/15, $\$ 91$

\section{ABSTRACTS}

La Cour européenne a rendu le 1er février 2018 sa décision quant à l'expulsion de M.A, condamné en France pour terrorisme, vers l'Algérie. Alors qu'un tel renvoi a pu constituer selon le requérant une violation de l'article 3 de la Convention mais aussi de l'article 34 du fait de son exécution expresse, l'intérêt majeur de cette affaire réside davantage dans le raisonnement juridique porté par la Cour que dans ses conclusions. En effet, il apparaît au fil des lignes une référence constante à l'affaire Daoudi, dont les faits de l'espèce sont fortement similaires, faisant de cette jurisprudence un modèle en matière d'expulsion vers l'Algérie. Par 
suite, s'établit peu à peu une véritable présomption de pays non-sûr pour cet Etat. Jurisprudence certes protectrice des droits des ressortissants de pays tiers, et néanmoins critiquable, l'affaire M.A contre France ne manque pas de contradictions et d'audace dont les origines tiennent sûrement à l'aspect purement symbolique du constat de violation de la Convention. Ainsi, reprenant tel un leitmotiv les conclusions du 3 décembre 2009, la jurisprudence M.A contre France et à travers elle l'affaire Daoudi, apparaissent comme une protection pour tous, mais surtout, et en l'espèce, pour personne.

\section{AUTHOR}

\section{ALMODIS PEYRE}

Etudiant du M2 Droit International Public de l'Université Lyon III 